\title{
Early treatment of skeletal open-bite malocclusion
}

\author{
Is early treatment of skeletal open-bite malocclusion effective?
}

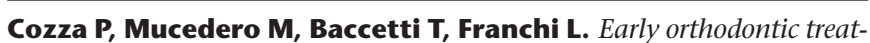
ment of skeletal open-bite malocclusion: a systematic review. Angle Orthod 2005; 75: 707-713

Data sources Studies were identified by searching the Cochrane Controlled Clinical Trials Register and Medline.

Study selection Randomised clinical trials (RCT), prospective and retrospective studies that had concurrent untreated as well as normal controls, and clinical trials that compared at least two treatment strategies but did not have any untreated or normal control group were included. The following were excluded: case series; descriptive studies; case reports; studies concerning treatment in the permanent dentition/ adult patients, surgically assisted treatment, treatment combined with extractions, treatment with full-fixed appliances; and discussion or debate articles.

Data extraction and synthesis Year of publication, study design, materials, dropouts, measurements, treatment time, success rate, decrease of open bite and divergence, side effects, costs, and authors' conclusions were recorded. Two independent reviewers assessed the articles independently and a quality evaluation and qualitative summary were performed.

Results A total of 1049 articles were identified of which seven were included in the review. No RCT of early treatment of anterior open bite have been performed. Two controlled clinical trials of early anterior open bite were identified, and these both indicated the effectiveness of treatment in the mixed dentition with headgear or functional appliances (or both). Most of the studies had serious problems with lack of power because of small sample size, bias and confounding variables; lack of method-error analysis or blinding in measurements; and deficient or lack of statistical methods.

Conclusions The quality of the studies was insufficient to allow any evidence-based conclusions to be drawn. RCT of sufficient sample size are still needed to determine which treatment is the most effective for early correction of skeletal open bite. Future studies should also include the assessment of long-term stability as well as an analysis of the cost and side-effects of the interventions.
Address for correspondence: Lorenzo Franchi, Dipartimento di

Odontostomatologia, Università degli Studi di Firenze, Via del Ponte di Mezzo, 4648, Firenze 50127, Italy. E-mail: 1.franchi@odonto.unifi.it

\section{Commentary}

This article addresses an important issue in early orthodontic treatment. The aim of this review was to find out if early treatment of open-bite is effective, which treatment modality is most effective, and if the result is stable. Unfortunately, the authors were not able to answer these questions because of a series of drawbacks in the studies reviewed.

The review was well-designed and properly performed, although there are two points that require clarification. The title is, "Early orthodontic treatment of skeletal open-bite malocclusion" and yet there was no discrimination between dental or skeletal open-bite in neither the search strategy nor the description of the results. Both terms were used in the review without clear definitions. (The review authors have since indicated that a majority of papers described treatment of malocclusions that presented with a combination of both dental and skeletal open-bite.) Secondly, "success rate" was given as one of the parameters for evaluating the results, but it was not clear how the authors defined the success rate, nor how the reviewed studies defined it. Is the presence of front contact a success? Or should it be the presence of vertical overlap of the front teeth?

In their inclusion criteria, the authors did not set any restrictions for sample size while noting in the Results that this was a major drawback. It seems that if sample size was included as one of the initial inclusion criteria, the conclusion could have been drawn without going into the details. Looking at the year of publication of the seven included studies, five are from 1992, one is from 1990 and one from 1983. It seems that the orthodontic field has not added new evidence to the literature for the past 14 years. Also related to the year of publication, it is understandable that ethical approval - a requirement for recent publications - was missing in all the reviewed studies.

A quick check in PubMed for systematic reviews or meta-analysis in orthodontics indicates that many of the results point to similar conclusions: "the results are inconclusive"; "there is no evidence or only a lower level of evidence", and, "randomised clinical trials, cost analysis and long-term studies are needed". Regarding all the reviews, one wonders if studies of this nature or such inconclusive reviews should be considered before the significance of their contribution to the orthodontic literature is evaluated. ${ }^{1}$

\section{Practice point}

The quality of studies in this area is insufficient to draw any evidence-based conclusions.

\section{Yijin Ren \\ Department of Orthodontics, University Medical Centre Groningen, Groningen, The Netherlands \\ 1. Papadopoulos MA. Meta-analysis in evidence-based orthodontics. Orthod Craniofac Res 2003; 6:112-126. \\ Evidence-Based Dentistry (2006) 7, 81-82. doi:10.1038/sj.ebd.6400450}

